\title{
Communication tools and sources of education and information: a national survey of rural and remote
} nurses

Julie Kosteniuk, PhD; Norma J. Stewart, PhD, RN: Erin C. Wilson, PhD, NP(F); Kelly L. Penz, PhD, RN; Ruth MartinMisener, PhD, NP; Debra G. Morgan, PhD, RN; Chandima Karunanayake, PhD; Martha L. P. MacLeod, PhD, RN

See end of article for authors' affiliations.

\begin{abstract}
Objective: This study examined accessibility of communication tools in the workplace, use of education to update nursing knowledge, and use of information to make specific decisions in practice among registered nurses (RNs) and licensed practical nurses (LPNs) in rural and remote communities in Canada.
\end{abstract}

Methods: Data were analyzed from the cross-sectional survey, "Nursing Practice in Rural and Remote Canada II," of regulated nurses practicing in all provinces and territories of Canada. Data were collected from April 2014 to August 2015.

Results: The survey was completed by 3,822 of 9,622 nurses ( $40 \%$ response), and the present analysis was conducted with a subsample of 2,827 nurses. High-speed Internet was the most accessible communication tool, and nurses used "online/electronic education" more often than "in-person education" to update their nursing knowledge. Internet searches were used more often than several other online/electronic sources to inform decision making. Compared to LPNs, RNs reported greater workplace access to most communication tools and greater use of online/electronic education as well as information sources in online/electronic and print formats. Compared to nurses in community-based health care and hospital settings, nurses in long-term care settings reported lower access to most communication tools, lower use of online/electronic and inperson education, and lower use of online/electronic information.

Conclusions: Access to continuing education and up-to-date information is important for effective patient care. This study points to a need for further research on the continuing education and information needs of rural and remote RNs and LPNs, and on their capacity to incorporate and apply new knowledge in practice.

\section{INTRODUCTION}

Three groups of nursing professionals are regulated to practice in Canada [1]: registered nurses (RNs), including nurse practitioners (NPs); licensed practical nurses (LPNs); and registered psychiatric nurses (RPNs). RNs and LPNs represent $71 \%$ and $28 \%$ of all regulated nurses in Canada, respectively [1], as well as the majority of regulated nurses in the United States [2]. RNs and LPNs in both countries are required to pass a national exam before licensure; however, licensing and regulatory oversight is provided by states, provinces, and territories [2-6]. Historically, practical nursing programs were created to train LPNs in a short period of time as a way to quickly fill a gap in the RN workforce during World War II [7]. Canadian provinces began to require a 4-year bachelor's degree for entry to RN practice as recently as 1998, except for Quebec, where a diploma is still the requirement $[1,8]$. Forty-five percent of Canadian RNs remain diploma prepared [9]. Educational preparation for RNs in the United States is similar, ranging from a diploma to a 4-year bachelor's degree [2]. 
Educational programs for LPNs in Canada, initially one year and now two years, have historically been shorter than those for RNs [10]. Similarly, a one-year program is required for LPN preparation in the United States [2]. Consequently, RNs are expected to have a more developed knowledgebase and clinical skills than LPNs, more advanced critical thinking skills, and a greater capacity for working autonomously and with populations with complex needs [11, 12].

Nevertheless, both RNs and LPNs are expected to build on their knowledgebases after initial training and to maintain their competencies [13].

In Canada, $10 \%$ of RNs [14] and 14\% of LPNs [15] practice in rural areas, where $17 \%$ of the population lives [16]. Compared to LPNs, RNs report broader responsibilities in their rural nursing practice that require more complex and analytical thinking related to several areas [17]. For instance, a greater proportion of rural RNs than LPNs report responsibility for independently making a nursing diagnosis (66\% versus $36 \%$ ), referring patients to other health care practitioners ( $48 \%$ versus $29 \%$ ), prescribing medication using protocols $(30 \%$ versus $12 \%)$, interpreting laboratory and diagnostic tests ( $46 \%$ versus $25 \%$ ), supervising or mentoring nursing colleagues (61\% versus $32 \%)$, and leading a unit or shift $(47 \%$ versus $31 \%)$.

The diversity and challenges in rural communities and work settings compel rural nurses to adapt quickly, think critically, and practice effectively despite restricted resources [18, 19], while applying advanced clinical judgment and skills that allow expanded practice roles [20-22]. Rural nurses must fill multiple roles and provide care to patients with varied acute and chronic conditions from diverse cultures and across all age groups [18, 23], often while practicing without nursing colleagues in the same setting [19]. Although rural nurses practice in varying degrees of professional isolation, there may be opportunities to collaborate with other health care professionals working in the same community or in neighboring communities and to draw support from these professionals [19, 24].

To ensure that rural nurses have the capacity to provide safe and effective care, and to maintain competence, it is important that they have opportunities to update their clinical knowledge and skills through continuing education programs [25]. Rural nurses require education that is relevant to their practice and patient population $[19,25]$.
Relevant continuing education has been shown to increase self-reported confidence, competence, and job satisfaction among rural nurses and to expand their range of practice [26]. However, studies have noted the limited availability of educational opportunities in rural workplaces [24, 27].

Urban-based learning opportunities pose geographical barriers for rural nurses that require organizational support and resources to be overcome, such as paid time, funding, relief staff, and formal requirements or incentives for continuing education [24, 27-30]. Although distance-based learning with synchronous and asynchronous webinars and virtual classrooms reduces the expense and time of travel $[25,31]$, organizations must still provide support (e.g., paid time or position coverage and back-up). Moreover, some rural communities in North America have lower access to high-speed Internet than urban centers [32,33], making distance-based learning challenging $[9,11]$. For instance, $52 \%$ and $61 \%$ of individuals in rural areas of Canada and the United States, respectively, have access to highspeed Internet, defined as a download speed of 25 megabits per second (Mbps) or higher [32, 33]. In comparison, $100 \%$ and $96 \%$ of those in urban centers of Canada and the United States, respectively, have high-speed Internet access.

Several studies of continuing education and information sources in rural nursing practice have centered exclusively on RNs (e.g., Winters et al. [24], O'Lynn et al. [34], Jukkala at el. [35], and Koessel et al. [36]). When studies included LPNs with other types of nurses in rural practice, most examined nurses as a single group rather than examining LPNs separately, and all of the studies considered questions such as needs or barriers to use rather than rural nurses' use of education or information for practice (e.g., Fairchild et al. [30], Carter-Templeton and $\mathrm{Wu}$ [37], Hodge et al. [38]). Given that the nursing responsibilities and education requirements for LPNs and RNs differ and that LPNs account for over one-quarter of the regulated nursing profession in Canada [1], separate examinations of RN and LPN education and information use are warranted.

Further, many studies of continuing education or information sources in rural nursing practice have focused either on single practice settings (e.g., Jukkala at el. [35], Carter-Templeton and Wu [37], Hodge et al. [38], Mills et al. [39], and Ortiz and Bushy [40]) or have included multiple settings but 
have not analyzed outcomes by setting (e.g., Winters et al. [24], Olade [29], O'Lynn et al. [34], Koschel et al. [41]). When studies have compared outcomes across rural practice settings, the outcomes have included learning needs or attitudes to evidencebased practice rather than nurses' use of education or information (e.g., Fairchild et al. [30] and Koessel et al. [36]).

Given how little is known about variations in education and information use by practice setting and type of rural nurse, the purpose of the present analysis was threefold. First, the authors investigated the accessibility of communication tools in the primary workplaces of rural and remote RNs and LPNs in Canada. Second, we examined the frequency with which rural and remote RNs and LPNs used education sources to update nursing knowledge and information sources to make specific decisions in nursing practice. Third, we analyzed the findings by registration status (RNs versus LPNs) and practice setting (community-based health care, hospital, and long-term care).

\section{METHODS}

\section{Design}

This project used data from the cross-sectional "Nursing Practice in Rural and Remote Canada II (RRNII)" survey of regulated nurses residing in rural and remote communities across all ten provinces and three territories of Canada. The RRNII survey replicated and extended the first "Nature of Nursing Practice in Rural and Remote Canada (RRNI)" cross-sectional survey [42].

MacLeod et al. provide further details about the RRNII study design and methods [43].

\section{Study population and sample}

For the RRNII survey, work postal codes or home postal codes, when work codes were unavailable, were used to systematically sample rural RNs, LPNs, and RPNs in all 10 Canadian provinces. All rural and remote NPs were also included in the sample. Communities across the 10 Canadian provinces with a core population of less than 10,000 people and a commuting segment of less than 50\% of the employed core, were considered rural [44]. All regulated nurses in the 3 territories were considered remote and were included in the sample.

\section{Data collection}

Using the Dillman method of persistent follow-up [45], data were collected by paper and online surveys in English and French from April 2014 to August 2015. A total of 10,072 nurses were sampled; 9,622 were eligible for the survey (i.e., practiced in a rural or remote community at the time of the survey and on leave for 6 months or less) and 450 were ineligible (e.g., worked in an urban area, address incorrect). A total 3,822 of 9,622 nurses completed a survey (response rate of $40 \%$ ), including 2,082 RNs, 1,370 LPNs, 163 NPs, and 207 RPNs.

The present analysis included 2,827 nurses: 1,646 RNs (excluded $n=436$ ) and 1,181 LPNs (excluded $n=189$ ). Nurses were included if they were employed in nursing and excluded if they were on leave or retired; held a primary position as an educator, researcher, consultant, or analyst; or were primarily employed in an educational institution, professional association or government, or workplace other than those described below. Also excluded from this analysis were NPs and RPNs, due to the small samples.

\section{Measures}

Outcome measures. Informed by previous studies [46-48], this study assessed several main outcomes (supplemental appendix). One outcome measure, "direct access to communication tools in the primary workplace for use in nursing practice," was assessed using 5 items (yes/no). All other outcomes were assessed with questions regarding how often nurses used specific sources of education and information. Higher scores indicated more frequent use, with all items scored on a 6-point scale from 1 (never), 2 (less than once a year), 3 (at least once a year), 4 (at least once a month), 5 (at least once a week), to 6 (daily). Outcomes measures of "online/electronic education use to update nursing knowledge" and "in-person education use to update nursing knowledge" were each assessed using 1 item. As part of this item, examples of education sources were provided to respondents and included in-service training, workplace education, continuing education, journal clubs, or nursing associations or colleges.

The outcome "online/electronic information use to make specific decisions in nursing practice" was measured with 7 items. A summated score was created by summing the scores for all 7 items, with total scores ranging from 7 to 42 and higher scores 
indicating more frequent use of online/electronic information. The Cronbach's alpha estimate for all 7 items was 0.84 . The outcome "print/paper information use to make specific decisions in nursing practice" consisted of 4 items. The scores of all 4 items were summed to create a summated score; more frequent use of print/paper information was reflected by higher scores. The Cronbach's alpha estimate for all 4 items was 0.78 . Lastly, frequent users of education or information were defined as nurses who used an education or information source daily or at least once a week and infrequent users were those who used a source at least once a month, at least once a year, less than once a year, or never.

Independent variables. The 8 independent demographic and professional practice variables consisted of sex (female or male); age; registration status (RN or LPN); highest nursing education credential (diploma, bachelor's, master's, or doctorate); primary position (staff nurse, nurse practitioner, clinical nurse specialist, or manager); living and working in same community (yes or no); and population of primary work community (under $1,000,1,000-9,999$, and 10,000 or over). Practice setting included 13 choices recoded to a single variable with 3 categories (community-based health care, hospital, and long-term care).

Nine practice issue measures were also included as independent variables. The scope of practice variable asked respondents to indicate whether they thought their roles were below, within, or above their registered or licensed scope of practice. The 4point work confidence item asked respondents to describe their levels of confidence in their work from 1 (extremely low) to 4 (extremely high). Scores on the single-item satisfaction with current nursing practice measure ranged from 1 (strongly disagree) to 5 (strongly agree), with higher scores indicating greater satisfaction.

Nine items were included from the Utrecht Work Engagement Scale [49], each scored from 0 (never/a few times a year) to 6 (always/everyday). Total scale scores ranged from 0 to 54 , and higher scores indicated greater work engagement. The original Organizational Commitment Scale [50] was adapted and reduced from 18 to 12 items, each scored from 1 (strongly disagree) to 7 (strongly agree). After reverse scoring 3 items, total scale scores ranged from 12 to 84 and higher scores indicated greater workplace commitment.
Three subscales of the Job Resources in Nursing (JRIN) Scale and 1 subscale of the Job Demands in Nursing Scale (JDIN) were included in this analysis [51]. The 3 JRIN subscales (staffing and time; technology; and training, professional development, and continuing education) and 1 JDIN subscale (preparedness/scope of practice) each consisted of 4 items that were scored from 1 (strongly disagree) to 5 (strongly agree), with subscale scores ranging from 4 to 20 and higher scores indicating a higher perceived level of the resource or demand (some JDIN-preparedness items required reverse scoring).

\section{Statistical analyses}

Statistical analysis was conducted with SPSS version 24.0. Descriptive statistics (frequency, mean, and standard deviation [SD]) were calculated by registration status (RNs and LPNs) and practice setting (community-based health care, hospital, and long-term care). Significant differences $(p<0.05)$ by registration status were identified with the $\chi^{2}$ test for nominal variables. Pairwise comparisons by practice setting were also examined with the $\chi^{2}$ test. We used 2-way analysis of variance (ANOVA) with 2 factors - registration status and practice setting - to examine main effects and interaction for 4 separate interval variables: online/electronic education to update nursing knowledge, in-person education to update nursing knowledge, summated online/electronic information to make specific decisions, and summated print information to make specific decisions. The Tukey post hoc test was used to examine pairwise differences across the 3 practice setting groups, for each of the 4 outcomes.

We selected the source used most often by nurses to update knowledge (online/electronic education) and the source used most often to make specific decisions in nursing practice (Internet search engines) to examine differences in practice issues according to frequency of use. Practice issues were compared for significant differences $(p<0.05)$ with the $\chi^{2}$ test for nominal variables and Student's $t$-test for interval variables across 2 groups (frequent and infrequent users) for RNs and LPNs separately.

\section{Ethics approval}

Approval was received by the university ethics boards of research team members in the provinces (British Columbia, Alberta, Saskatchewan, Ontario, Quebec, and Nova Scotia) and research licensing boards in the Northwest Territories and Nunavut. 


\section{RESULTS}

\section{Sample characteristics}

Respondents were, on average, 46.6 years of age $(\mathrm{SD}=11.5$, range $=20-84)$. RNs were 47.0 years of age on average $(\mathrm{SD}=11.6$, range $=22-84)$, and LPNs were 46.1 years $(S D=11.3$, range $=20-70)$. The majority of respondents were female, worked in the same community where they lived, and worked in communities with populations less than 10,000 (Table 1). A similar proportion of RNs held a bachelor's degree as their highest credential (48.4\%) as held a diploma (49.4\%), and nearly all LPNs held a diploma as their highest credential (99.6\%). Most RNs and LPNs were staff nurses, and the majority were employed in hospitals.

\section{Direct access to communication tools in workplace}

Overall, the most accessible workplace communication tool was high-speed Internet (86.3\%) and the least accessible was web conferencing such as Skype or WebEx (30.8\%) (Table 2). Most nurses reported that they had access to electronic communication among providers such as email or text $(80.2 \%)$, as well as teleconference $(70.2 \%)$ and videoconference tools (54.4\%). Access varied by nurse group, with direct workplace access to most communication tools reported by a greater proportion of RNs than LPNs. Group differences in access to high-speed Internet were not reported.

Direct workplace access to communication tools also varied by practice setting (Tables 2 and 3 ). Access to high-speed Internet was greater in hospital settings than community-based health care and long-term care settings. Nurses in community-based health care settings had greater access to electronic communication among providers and to web conferencing than those in other settings. Moreover, access to electronic communication between providers was higher in hospitals than long-term care settings. Teleconference and videoconference access was lower in long-term care settings compared to other settings.

\section{Use of education sources to update nursing knowledge}

Nurses overall used online/electronic education more often than in-person education to update nursing knowledge (Table 4). Two-way ANOVA analyses showed that registration status had a main effect on the use of online/electronic education to update nursing knowledge (Table 5), with use higher by RNs than LPNs. Practice setting had main effects on the use of both online/electronic and inperson education to update nursing knowledge. Post hoc tests showed more frequent use of online/electronic education in community-based health care compared to hospital and long-term care settings, as well as greater use in hospital compared to long-term care. Furthermore, in-person education was used more often in community-based health care and hospital settings than in long-term care settings. There were no significant interaction effects on the use of either education source.

\section{Use of information sources to make specific decisions} in nursing practice

As shown in Table 4, the rank ordered frequency of online/electronic information use for the purpose of making specific decisions in practice was similar between RNs and LPNs. In descending order, the rankings were: Internet search engines (e.g., Google, Yahoo); policies, protocols, standards, or regulatory tools (hereafter referred to as policies or protocols); clinical practice guidelines; nursing or medical textbooks; nursing or medical journals; practice support resources (e.g., NurseOne, UpToDate, eMedicine); and research databases (e.g., CINAHL, Medline, PubMed). The rank ordered frequency of print use was also similar between both nurse groups, following a descending order: policies or protocols, clinical practice guidelines, nursing or medical textbooks, and nursing or medical journals.

Two-way ANOVA analyses indicated that registration status had a main effect on the use of both online/electronic information and print information to make specific decisions (Table 5), with summated scores for both outcomes higher among RNs than LPNs. Practice setting also had a main effect on both outcomes, with Tukey post hoc tests showing a higher summated score on online/electronic information in community-based health care compared to other settings and in hospitals compared to long-term care workplaces (Table 5). Likewise, the summated score for print information use was higher in community-based health care compared to other settings. There were no significant interaction effects on the total use of all sources, regardless of format. 
Table 1 Characteristics of respondents

\begin{tabular}{|c|c|c|c|c|c|c|}
\hline \multirow[b]{2}{*}{ Characteristics } & \multicolumn{2}{|c|}{ Total } & \multicolumn{2}{|c|}{ Registered nurse (RN) } & \multicolumn{2}{|c|}{$\begin{array}{l}\text { Licensed practical nurse } \\
\text { (LPN) }\end{array}$} \\
\hline & $\mathbf{n}$ & $(\%)$ & $\mathbf{n}$ & $(\%)$ & $\mathbf{n}$ & $(\%)$ \\
\hline \multicolumn{7}{|l|}{ Registration status } \\
\hline RN & 1,646 & $(58.2 \%)$ & 1,646 & $(100.0 \%)$ & 0 & $(-)$ \\
\hline LPN & 1,181 & $(41.8 \%)$ & 0 & $(-)$ & 1,181 & $(100.0 \%)$ \\
\hline Total & 2,827 & & 1,646 & & 1,181 & \\
\hline \multicolumn{7}{|l|}{ Sex } \\
\hline Male & 166 & $(6.0 \%)$ & 99 & $(6.1 \%)$ & 67 & $(5.9 \%)$ \\
\hline Female & 2,592 & $(94.0 \%)$ & 1,515 & $(93.9 \%)$ & 1,077 & $(94.1 \%)$ \\
\hline Total & 2,758 & & 1,614 & & 1,144 & \\
\hline \multicolumn{7}{|l|}{ Highest attained nursing education } \\
\hline Diploma & 1,973 & $(70.5 \%)$ & 802 & $(49.4 \%)$ & 1,171 & $(99.6 \%)$ \\
\hline Bachelor's & 790 & $(28.2 \%)$ & 785 & $(48.4 \%)$ & 5 & $(0.4 \%)$ \\
\hline Master's & 34 & $(1.2 \%)$ & 34 & $(2.1 \%)$ & 0 & $(-)$ \\
\hline Doctorate & 1 & $(<0.1 \%)$ & 1 & $(<0.1 \%)$ & 0 & $(-)$ \\
\hline Total & 2,798 & & 1,622 & & 1,176 & \\
\hline \multicolumn{7}{|l|}{ Primary position } \\
\hline Staff nurse & 2,464 & $(87.2 \%)$ & 1,334 & $(81.0 \%)$ & 1,130 & $(95.7 \%)$ \\
\hline Nurse practitioner & 116 & $(4.1 \%)$ & 101 & $(6.1 \%)$ & 15 & $(1.3 \%)$ \\
\hline Manager & 247 & $(8.7 \%)$ & 211 & $(12.8 \%)$ & 36 & $(3.0 \%)$ \\
\hline Total & 2,827 & & 1,646 & & 1,181 & \\
\hline \multicolumn{7}{|l|}{ Practice setting } \\
\hline Community-based health care & 746 & $(26.4 \%)$ & 595 & $(36.1 \%)$ & 151 & $(12.8 \%)$ \\
\hline Hospital & 1,454 & $(51.4 \%)$ & 863 & $(52.4 \%)$ & 591 & $(50.0 \%)$ \\
\hline Long-term care & 627 & $(22.2 \%)$ & 188 & $(11.4 \%)$ & 439 & $(37.2 \%)$ \\
\hline Total & 2,827 & & 1,646 & & 1,181 & \\
\hline Live and work in same community & 1,578 & $(56.9 \%)$ & 966 & $(59.7 \%)$ & 612 & $(53.0 \%)$ \\
\hline Total & 2,772 & & 1,618 & & 1,155 & \\
\hline \multicolumn{7}{|l|}{$\begin{array}{l}\text { Population of primary work } \\
\text { community }\end{array}$} \\
\hline Under 1,000 & 368 & $(13.5 \%)$ & 230 & $(14.4 \%)$ & 138 & $(12.2 \%)$ \\
\hline $1,000-9,999$ & 1,523 & $(55.8 \%)$ & 861 & $(54.0 \%)$ & 662 & $(58.4 \%)$ \\
\hline 10,000 and over & 836 & $(30.7 \%)$ & 503 & $(31.6 \%)$ & 333 & $(29.4 \%)$ \\
\hline Total & 2,727 & & 1,594 & & 1,133 & \\
\hline
\end{tabular}

Note: Sample sizes vary due to missing values. 
DOI: dx.doi.org/10.5195/jmla.2019.632

Table 2 Direct access to communication tools, by registration status and practice setting

\begin{tabular}{|c|c|c|c|c|c|c|c|c|c|c|c|c|c|c|c|c|}
\hline \multirow{3}{*}{$\begin{array}{c}\text { Communication } \\
\text { tool }\end{array}$} & \multicolumn{8}{|c|}{ Registration status } & \multicolumn{8}{|c|}{ Practice setting } \\
\hline & \multicolumn{2}{|c|}{ Total } & \multicolumn{2}{|c|}{$\mathbf{R N}$} & \multicolumn{2}{|c|}{ LPN } & \multirow{2}{*}{$\begin{array}{l}\text { Chi- } \\
\text { square }\end{array}$} & \multirow{2}{*}{$\begin{array}{c}p \\
\text { value }\end{array}$} & \multicolumn{2}{|c|}{$\begin{array}{l}\text { Community- } \\
\text { based health } \\
\text { care }\end{array}$} & \multicolumn{2}{|c|}{ Hospital } & \multicolumn{2}{|c|}{$\begin{array}{c}\text { Long-term } \\
\text { care }\end{array}$} & \multirow{2}{*}{$\begin{array}{l}\text { Chi- } \\
\text { square }\end{array}$} & \multirow{2}{*}{$\begin{array}{c}p \\
\text { value }\end{array}$} \\
\hline & n & $(\%)$ & $\mathbf{n}$ & $(\%)$ & $\mathbf{n}$ & $(\%)$ & & & $\mathbf{n}$ & $(\%)$ & $\mathbf{n}$ & $(\%)$ & n & $(\%)$ & & \\
\hline $\begin{array}{l}\text { High-speed } \\
\text { Internet }\end{array}$ & 2,439 & $(86.3 \%)$ & 1,436 & $(87.2 \%)$ & 1,003 & $(84.9 \%)$ & 3.11 & 0.078 & 627 & $(84.0 \%)$ & 1,293 & $(88.9 \%)$ & 519 & $(82.8 \%)$ & 18.25 & $<0.001$ \\
\hline $\begin{array}{l}\text { Electronic } \\
\text { communication } \\
\text { among providers }\end{array}$ & 2,266 & $(80.2 \%)$ & 1,391 & $(84.5 \%)$ & 875 & $(74.1 \%)$ & 46.92 & $<0.001$ & 657 & $(88.1 \%)$ & 1,176 & $(80.9 \%)$ & 433 & $(69.1 \%)$ & 78.39 & $<0.001$ \\
\hline Teleconference & 1,985 & $(70.2 \%)$ & 1,267 & $(77.0 \%)$ & 718 & $(60.8 \%)$ & 86.06 & $<0.001$ & 573 & $(76.8 \%)$ & 1,072 & $(73.7 \%)$ & 340 & $(54.2 \%)$ & 100.73 & $<0.001$ \\
\hline Videoconference & 1,537 & $(54.4 \%)$ & 1,009 & $(61.3 \%)$ & 528 & $(44.7 \%)$ & 76.31 & $<0.001$ & 448 & $(60.1 \%)$ & 868 & $(59.7 \%)$ & 221 & $(35.2 \%)$ & 118.76 & $<0.001$ \\
\hline Web conferencing & 870 & $(30.8 \%)$ & 589 & $(35.8 \%)$ & 281 & $(23.8 \%)$ & 46.4 & $<0.001$ & 304 & $(40.8 \%)$ & 407 & $(28.0 \%)$ & 159 & $(25.4 \%)$ & 48.77 & $<0.001$ \\
\hline
\end{tabular}

Note: Total $(n=2,827)$, RN ( $=1,646)$, and LPN ( $=1,181)$; community-based health care $(n=746)$, hospital $(n=1,454)$, and long-term care $(n=627)$. $p$ values $<0.05$ are bolded.

Table 3 Direct access to communication tools, by practice setting

\begin{tabular}{|c|c|c|c|c|c|c|}
\hline \multirow[b]{2}{*}{ Communication tool } & \multicolumn{2}{|c|}{$\begin{array}{c}\text { Community-based health care vs. } \\
\text { hospital }\end{array}$} & \multicolumn{2}{|c|}{$\begin{array}{l}\text { Community-based health } \\
\text { care vs. long-term care }\end{array}$} & \multicolumn{2}{|c|}{$\begin{array}{c}\text { Hospital vs. long-term } \\
\text { care }\end{array}$} \\
\hline & Chi-square & $p$ value & Chi-square & $p$ value & Chi-square & $p$ value \\
\hline High-speed Internet & 10.57 & 0.001 & 0.4 & 0.527 & 14.73 & $<0.001$ \\
\hline Electronic communication among providers & 18.34 & $<0.001$ & 75.24 & $<0.001$ & 34.91 & $<0.001$ \\
\hline Teleconference & 2.48 & 0.115 & 77.99 & $<0.001$ & 76.3 & $<0.001$ \\
\hline Videoconference & 0.026 & 0.872 & 83.91 & $<0.001$ & 104.99 & $<0.001$ \\
\hline Web conferencing & 36.69 & $<0.001$ & 36.11 & $<0.001$ & 1.53 & 0.216 \\
\hline
\end{tabular}

Note: Total $(n=2,827)$, community-based health care $(n=746)$, hospital $(n=1,454)$, and long-term care $(n=627) . p$ values $<0.05$ are bolded. 
Table 4 Direct access to communication tools, by practice setting

\begin{tabular}{|c|c|c|c|c|c|c|c|c|c|c|c|c|}
\hline \multirow[b]{3}{*}{ Education or information source } & \multirow{2}{*}{\multicolumn{2}{|c|}{ Total }} & \multicolumn{4}{|c|}{ Registration status } & \multicolumn{6}{|c|}{ Practice setting } \\
\hline & & & \multicolumn{2}{|c|}{$\mathbf{R N}$} & \multicolumn{2}{|c|}{ LPN } & \multicolumn{2}{|c|}{$\begin{array}{c}\text { Community-based } \\
\text { health care }\end{array}$} & \multicolumn{2}{|c|}{ Hospital } & \multicolumn{2}{|c|}{ Long-term care } \\
\hline & $\mathbf{M}$ & (SD) & $\mathbf{M}$ & (SD) & $\mathbf{M}$ & (SD) & $\mathbf{M}$ & (SD) & $\mathbf{M}$ & (SD) & $\mathbf{M}$ & (SD) \\
\hline \multicolumn{13}{|l|}{ Education to update nursing knowledge } \\
\hline Online/electronic & 4.0 & $(1.2)$ & 4.2 & $(1.1)$ & 3.7 & $(1.3)$ & 4.3 & $(1.1)$ & 4.0 & $(1.2)$ & 3.6 & $(1.3)$ \\
\hline In-person & 3.6 & $(1.1)$ & 3.7 & $(1.1)$ & 3.5 & $(1.1)$ & 3.7 & $(1.2)$ & 3.6 & $(1.0)$ & 3.5 & $(1.1)$ \\
\hline \multicolumn{13}{|l|}{$\begin{array}{l}\text { Online/electronic information to make } \\
\text { specific decisions in nursing practice }\end{array}$} \\
\hline Internet search engines (e.g., Google) & 4.8 & $(1.3)$ & 4.9 & $(1.2)$ & 4.6 & $(1.4)$ & 5.0 & $(1.1)$ & 4.8 & $(1.2)$ & 4.3 & $(1.5)$ \\
\hline $\begin{array}{l}\text { Policies, protocols, standards, or } \\
\text { regulatory tools }\end{array}$ & 4.4 & $(1.5)$ & 4.7 & $(1.3)$ & 4.1 & $(1.5)$ & 4.7 & $(1.3)$ & 4.5 & $(1.4)$ & 3.9 & $(1.6)$ \\
\hline Clinical practice guidelines & 4.1 & $(1.5)$ & 4.4 & $(1.4)$ & 3.8 & $(1.5)$ & 4.5 & $(1.4)$ & 4.2 & $(1.4)$ & 3.7 & $(1.6)$ \\
\hline Nursing/medical textbooks & 3.4 & $(1.5)$ & 3.5 & $(1.4)$ & 3.4 & $(1.5)$ & 3.6 & $(1.5)$ & 3.4 & $(1.4)$ & 3.3 & $(1.5)$ \\
\hline Nursing/medical journals & 3.3 & $(1.4)$ & 3.5 & $(1.3)$ & 3.1 & $(1.5)$ & 3.7 & $(1.3)$ & 3.2 & $(1.4)$ & 3.1 & $(1.5)$ \\
\hline $\begin{array}{l}\text { Practice support resources (e.g., } \\
\text { NurseOne) }\end{array}$ & 3.2 & $(1.8)$ & 3.4 & $(1.8)$ & 2.8 & $(1.8)$ & 3.6 & $(1.8)$ & 3.2 & $(1.8)$ & 2.7 & $(1.6)$ \\
\hline Research databases (e.g., CINAHL) & 2.7 & $(1.6)$ & 2.9 & $(1.6)$ & 2.5 & $(1.7)$ & 3.1 & $(1.6)$ & 2.7 & $(1.6)$ & 2.4 & $(1.6)$ \\
\hline $\begin{array}{l}\text { Summated online/electronic information } \\
\text { use }\end{array}$ & 26.0 & $(7.5)$ & 27.1 & $(6.9)$ & 24.3 & $(7.9)$ & 27.6 & $(7.0)$ & 25.5 & $(7.3)$ & 22.9 & $(8.1)$ \\
\hline \multicolumn{13}{|l|}{$\begin{array}{l}\text { Print information to make specific } \\
\text { decisions in nursing practice }\end{array}$} \\
\hline $\begin{array}{l}\text { Policies, protocols, standards, or } \\
\text { regulatory tools }\end{array}$ & 4.3 & $(1.4)$ & 4.4 & $(1.4)$ & 4.1 & $(1.4)$ & 4.4 & $(1.4)$ & 4.3 & $(1.5)$ & 4.2 & $(1.4)$ \\
\hline Clinical practice guidelines & 4.0 & $(1.5)$ & 4.1 & $(1.5)$ & 3.8 & $(1.5)$ & 4.2 & $(1.5)$ & 3.9 & $(1.5)$ & 3.9 & $(1.5)$ \\
\hline Nursing/medical textbooks & 3.5 & $(1.4)$ & 3.5 & $(1.4)$ & 3.5 & $(1.4)$ & 3.7 & $(1.4)$ & 3.3 & $(1.4)$ & 3.6 & $(1.3)$ \\
\hline Nursing or medical journals & 3.1 & $(1.4)$ & 3.2 & $(1.4)$ & 3.0 & (1.4) & 3.3 & $(1.4)$ & 3.0 & $(1.4)$ & 3.2 & $(1.4)$ \\
\hline Summated print information use & 14.8 & $(4.5)$ & 15.2 & $(4.4)$ & 14.3 & $(4.6)$ & 15.3 & $(4.5)$ & 14.3 & $(4.5)$ & 14.7 & $(4.5)$ \\
\hline
\end{tabular}

Note: $\mathrm{M}=$ mean; $\mathrm{SD}=$ standard deviation. Scoring for single items: $1=$ never, $2=$ less than once a year, $3=$ at least once $a$ year, $4=$ at least once $a$ month, $5=a t$ least once a week, $6=$ daily. Samples vary due to missing cases: total ( $n=2,505-2,723), R N(n=1,489-1,592)$, LPN ( $n=1,016$ to 1,132$)$; community-based health care $(n=661-720)$, hospital $(n=1,290-1,403)$, and long-term care ( $n=554-$

605). $p$ values $<0.05$ are bolded. 
Table 5 Use of education and information, two-way analysis of variance (ANOVA) results for registration status and practice setting main effects and interaction

\begin{tabular}{|c|c|c|c|c|c|c|c|c|c|c|c|c|c|c|c|c|}
\hline & \multicolumn{4}{|c|}{$\begin{array}{c}\text { Online/electronic education to } \\
\text { update nursing knowledge }\end{array}$} & \multicolumn{4}{|c|}{$\begin{array}{c}\text { In-person education to update } \\
\text { nursing knowledge }\end{array}$} & \multicolumn{4}{|c|}{$\begin{array}{c}\text { Summated online/electronic } \\
\text { information use to make specific } \\
\text { decisions }\end{array}$} & \multicolumn{4}{|c|}{$\begin{array}{l}\text { Summated print information use } \\
\text { to make specific decisions }\end{array}$} \\
\hline & $d f$ & $F$ & $p$ & $\eta 2$ & $d f$ & $F$ & $p$ & $\eta^{2}$ & $d f$ & $F$ & $p$ & $\eta 2$ & $d f$ & $F$ & $p$ & $\eta 2$ \\
\hline \multicolumn{17}{|l|}{$\begin{array}{l}\text { Between } \\
\text { subjects }\end{array}$} \\
\hline $\begin{array}{l}\text { Registration } \\
\text { status }\end{array}$ & 1 & 62.77 & $<0.001$ & 0.023 & 1 & 3.31 & 0.069 & 0.001 & 1 & 30.83 & $<0.001$ & 0.012 & 1 & 22.76 & $<0.001$ & 0.009 \\
\hline $\begin{array}{l}\text { Practice } \\
\text { setting }\end{array}$ & 2 & 17.57 & $<0.001$ & 0.013 & 2 & 3.61 & 0.027 & 0.003 & 2 & 29.08 & $<0.001$ & 0.023 & 2 & 6.31 & 0.002 & 0.005 \\
\hline $\begin{array}{l}\text { Registration } \\
\text { status } \times \\
\text { Practice } \\
\text { setting }\end{array}$ & 2 & 1.79 & 0.167 & 0.001 & 2 & 1.17 & 0.312 & 0.001 & 2 & 0.301 & 0.74 & $<0.001$ & 2 & 0.73 & 0.482 & 0.001 \\
\hline Within-group & & & & & & & & & & & & & & & & \\
\hline $\begin{array}{l}\text { Mean square } \\
\text { b. Pairwise } \\
\text { comparisons }\end{array}$ & 2,706 & -1.39 & & & 2,717 & -1.17 & & & 2,499 & -52.37 & & & 2,735 & -20.17 & & \\
\hline $\begin{array}{l}\text { Community- } \\
\text { based } \\
\text { primary } \\
\text { health care } \\
\text { vs. hospital }\end{array}$ & & & $<0.001$ & & & & & 0.726 & & & $<0.001$ & & & & $<0.001$ & \\
\hline $\begin{array}{l}\text { Community- } \\
\text { based } \\
\text { primary } \\
\text { health care } \\
\text { vs. long- } \\
\text { term care }\end{array}$ & & & $<0.001$ & & & & & 0.003 & & & $<0.001$ & & & & 0.049 & \\
\hline $\begin{array}{l}\text { Hospital vs. } \\
\text { long-term } \\
\text { care }\end{array}$ & & & $<0.001$ & & & & & 0.007 & & & $<0.001$ & & & & 0.125 & \\
\hline
\end{tabular}

Note: Results analyzed by two-way ANOVA followed by Tukey test. Values enclosed in parentheses represent mean square errors. $p$ values $<0.05$ are bolded. 


\section{Practice issues}

To determine whether practice issues varied by the frequency with which certain sources were used, we identified the education source used most often to update nursing knowledge (online/electronic) and the information source used most often to make specific decisions in nursing practice (Internet search engines). Among RNs, frequent users of online/electronic education to update knowledge and frequent users of Internet searches to make specific decisions in practice demonstrated greater work engagement than infrequent users (Table 6). Further, frequent users of Internet searches to make decisions in practice indicated higher levels of technology as a practice resource than infrequent users.

Among LPNs, frequent users of online/electronic education to update knowledge indicated higher levels of practice resources related to technology and training or professional development than infrequent users (Table 7). Compared to infrequent users of Internet searches to make decisions in practice, frequent users reported higher levels of work engagement and technology as a practice resource.

\section{DISCUSSION}

Our study found a number of patterns in access to communication tools and use of education and information among rural and remote nurses. First, direct workplace access to five communication tools followed similar patterns for RNs and LPNs, with high-speed Internet the most accessible and web conferencing the least accessible tools. Additionally, both nurse groups more often drew on online/electronic than in-person education sources to update their nursing knowledge. Finally, the pattern of information use for decision making in nursing practice was similar across both nurse groups: Internet searches were most popular and research databases least popular in terms of online/electronic information, and policies or protocols were most popular and nursing or medical journals least popular in terms of print information.

Our finding that high-speed Internet was accessible to $86 \%$ of rural and remote nurses overall corresponds to previous studies that found a considerable majority of rural RNs have workplace Internet access [24, 34]. However, it is worth noting that 1 in 10 nurses in our study lacked workplace access to high-speed Internet. Further, web conferencing, an important tool for synchronous online learning [52], was directly accessible in a small minority of workplaces (31\% overall). Web conferencing tools were not defined for respondents in our study; however, the Skype and WebEx applications were provided as examples. Although taking part in web conferences generally requires little more than Internet access in terms of technology, it is also essential for participation that users have adequate time, adequate workspace, and a reliable Internet connection. Communication technology can reduce the professional and social isolation of rural nurses [31], address the challenges of travel to access urban-based in-person education [53], and support skill development through online learning [25], but these tools must first be accessible to nurses in their workplaces.

The higher use of online/electronic education than in-person education among rural and remote nurses in the present analysis suggests that online/electronic education formats are more accessible and/or relevant than face-to-face formats. A recent US study of rural hospital nurses, mainly RNs, found that nurses preferred in-person education, with online courses second in choice over other methods such as self-instruction and videoconferencing [38]. However, an early US study of mainly hospital-based rural RNs found that budget cuts meant fewer in-service opportunities, and external face-to-face education required travel to a city [24]. While in-person formats may be preferred, web-based formats alone or in conjunction with a face-to-face component [26] are perhaps more accessible than face-to-face alone, require less travel, may be more convenient and cost-efficient, and offer a range of educational opportunities. Slow Internet connections and lack of topic relevance, in addition to heavy workloads in understaffed workplaces, are barriers that remain to be addressed when offering online continuing education to rural nurses $[26,28]$.

The online/electronic and print information sources that rural and remote RNs and LPNs in this study reported using most and least often warrant closer examination. Considering online/electronic sources, Internet search engines were used most often, followed by policies or protocols, clinical practice guidelines, textbooks, journals, practice 
Table 6 Practice issues among registered nurses (RNs) by frequency of online/electronic education and Internet search engine use

\begin{tabular}{|c|c|c|c|c|c|c|c|c|c|c|}
\hline \multirow[b]{2}{*}{ Practice issue } & \multicolumn{5}{|c|}{ Online/electronic education to update nursing knowledge } & \multicolumn{5}{|c|}{$\begin{array}{l}\text { Internet search engines to make specific decisions in nursing } \\
\text { practice }\end{array}$} \\
\hline & \multicolumn{2}{|c|}{ Frequent use } & \multicolumn{2}{|c|}{ Infrequent use } & \multirow[t]{2}{*}{$\begin{array}{c}p \\
\text { value }\end{array}$} & \multicolumn{2}{|c|}{ Frequent use } & \multicolumn{2}{|c|}{ Infrequent use } & \multirow[t]{2}{*}{$p$ value } \\
\hline & $\mathbf{n}$ & $(\%)$ & $\mathbf{n}$ & $(\%)$ & & $\mathbf{n}$ & $(\%)$ & $\mathbf{n}$ & $(\%)$ & \\
\hline $\begin{array}{l}\text { Current role is within or above } \\
\text { registered or licensed scope of } \\
\text { practice }\end{array}$ & 580 & $(94.3 \%)$ & 915 & $(94.6 \%)$ & 0.821 & 1,066 & $(94.4 \%)$ & 423 & $(94.65)$ & 1 \\
\hline \multirow{2}{*}{$\begin{array}{l}\text { Work confidence (somewhat or } \\
\text { extremely high) }\end{array}$} & 587 & $(95.4 \%)$ & 923 & $(95.7 \%)$ & 0.801 & 1,078 & $(95.7 \%)$ & 426 & $(95.5 \%)$ & 0.892 \\
\hline & $\mathbf{M}$ & $\begin{array}{c}\mathrm{SD} \\
\text { (range) }\end{array}$ & $\mathbf{M}$ & $\begin{array}{c}\mathrm{SD} \\
\text { (range) }\end{array}$ & & $\mathbf{M}$ & SD (range) & $\mathbf{M}$ & SD (range) & \\
\hline $\begin{array}{l}\text { Satisfaction with current nursing } \\
\text { practice }\end{array}$ & $4.0 \pm 0.8$ & $(1-5)$ & $4.0+0.8$ & $(1-5)$ & 0.178 & $4.0+0.8$ & $(1-5)$ & $3.9+0.8$ & $(1-5)$ & 0.156 \\
\hline Work engagement & $40.0 \pm 9.0$ & $(9-54)$ & $37.6 \pm 9.4$ & $(0-54)$ & $<0.001$ & $38.9 \pm 9.1$ & $(2-54)$ & $37.5+9.8$ & $(0-54)$ & 0.006 \\
\hline Organizational commitment & $51.1 \pm 11.6$ & $(12-84)$ & $51.6 \pm 10.6$ & $(14-84)$ & 0.385 & $51.1 \pm 11.1$ & $(12-84)$ & $52.1 \pm 10.6$ & $(14-84)$ & 0.091 \\
\hline $\begin{array}{l}\text { Practice resource: staffing and } \\
\text { time }\end{array}$ & $11.8 \pm 3.7$ & $(4-20)$ & $11.7 \pm 3.6$ & $(4-20)$ & 0.68 & $11.8 \pm 3.6$ & $(4-20)$ & $11.8 \pm 3.6$ & $(4-20)$ & 0.901 \\
\hline Practice resource: technology & $13.4+3.5$ & $(4-20)$ & $13.2 \pm 3.2$ & $(4-20)$ & 0.18 & $13.4 \pm 3.3$ & $(4-20)$ & $13.0 \pm 3.4$ & $(4-20)$ & 0.047 \\
\hline $\begin{array}{l}\text { Practice resource: training, } \\
\text { professional development, and } \\
\text { continuing education }\end{array}$ & $12.8+3.6$ & $(4-20)$ & $12.5+3.5$ & $(4-20)$ & 0.13 & $12.6+3.6$ & $(4-20)$ & $12.6+3.4$ & $(4-20)$ & 0.863 \\
\hline $\begin{array}{l}\text { Practice demand: preparedness } \\
\text { or scope of practice }\end{array}$ & $7.8 \pm 2.1$ & $(4-17)$ & $7.8 \pm 1.9$ & $(4-16)$ & 0.604 & $7.8 \pm 2.1$ & $(4-17)$ & $7.9 \pm 1.8$ & $(4-16)$ & 0.356 \\
\hline
\end{tabular}

Note: $p$ values calculated by $\chi^{2}$ test for nominal variables and Student's $t$-test for interval variables. $p$ values $<0.05$ are bolded. Samples vary due to missing cases: Online/electronic education frequent use ( $n=577-615)$, infrequent use $(n=895-968)$; Internet search engines frequent use $(n=1,063-1,131)$, infrequent use $(n=404-448)$. 
Table 7 Practice issues among licensed practical nurses (LPNs) by frequency of online/electronic education and Internet search engine use

\begin{tabular}{|c|c|c|c|c|c|c|c|c|c|c|}
\hline \multirow[b]{2}{*}{ Practice issue } & \multicolumn{5}{|c|}{ Online/electronic education to update nursing knowledge } & \multicolumn{5}{|c|}{$\begin{array}{l}\text { Internet search engines to make specific decisions in nursing } \\
\text { practice }\end{array}$} \\
\hline & \multicolumn{2}{|c|}{ Frequent use } & \multicolumn{2}{|c|}{ Infrequent use } & \multirow[t]{2}{*}{$\begin{array}{c}p \\
\text { value }\end{array}$} & \multicolumn{2}{|c|}{ Frequent use } & \multicolumn{2}{|c|}{ Infrequent use } & \multirow[t]{2}{*}{$p$ value } \\
\hline & $\mathbf{n}$ & $(\%)$ & $\mathrm{n}$ & $(\%)$ & & $\mathrm{n}$ & $(\%)$ & $\mathbf{n}$ & $(\%)$ & \\
\hline $\begin{array}{l}\text { Scope of practice (current role is } \\
\text { within or above registered or } \\
\text { licensed scope of practice }\end{array}$ & 225 & $(83.0 \%)$ & 693 & $(82.0 \%)$ & 0.784 & 584 & $(82.0 \%)$ & 329 & $(81.6 \%)$ & 0.872 \\
\hline \multirow{2}{*}{$\begin{array}{l}\text { Work confidence (somewhat or } \\
\text { extremely high) }\end{array}$} & 253 & $(94.1 \%)$ & 784 & $(93.0 \%)$ & 0.675 & 663 & $(93.5 \%)$ & 370 & $(92.0 \%)$ & 0.393 \\
\hline & $\mathbf{M}$ & $\begin{array}{c}\text { SD } \\
\text { (range) }\end{array}$ & $\mathbf{M}$ & $\begin{array}{c}\text { SD } \\
\text { (range) }\end{array}$ & & $\mathbf{M}$ & SD (range) & $\mathbf{M}$ & SD (range) & \\
\hline $\begin{array}{l}\text { Satisfaction with current nursing } \\
\text { practice }\end{array}$ & $3.8+0.9$ & $(1-5)$ & $3.8+0.8$ & $(1-5)$ & 0.825 & $3.8+0.9$ & $(1-5)$ & $3.9+0.8$ & $(1-5)$ & 0.164 \\
\hline Work engagement & $38.7+9.3$ & $(5-54)$ & $37.8+9.6$ & $(0-54)$ & 0.164 & $38.5+9.4$ & $(0-54)$ & $37.1+9.7$ & $(7-54)$ & 0.017 \\
\hline Organizational commitment & $53.6 \pm 11.0$ & $(27-81)$ & $52.9 \pm 10.4$ & $(18-84)$ & 0.34 & $53.2 \pm 10.7$ & $(18-81)$ & $52.9 \pm 10.3$ & $(24-84)$ & 0.639 \\
\hline $\begin{array}{l}\text { Practice resource: staffing and } \\
\text { time }\end{array}$ & $11.5+3.8$ & $(4-20)$ & $11.0+3.4$ & $(4-20)$ & 0.068 & $11.1+3.6$ & $(4-20)$ & $11.1+3.4$ & $(4-20)$ & 0.864 \\
\hline Practice resource: technology & $13.7 \pm \pm+3.2$ & $(4-20)$ & $13.1+3.0$ & $(4-20)$ & 0.014 & $13.5+3.0$ & $(4-20)$ & $12.8+3.1$ & $(4-20)$ & 0.001 \\
\hline $\begin{array}{l}\text { Practice resource: training, } \\
\text { professional development, and } \\
\text { continuing education }\end{array}$ & $13.1 \pm 3.3$ & $(4-20)$ & $12.4+3.4$ & $(4-20)$ & 0.003 & $12.7 \pm 3.4$ & $(4-20)$ & $12.4+3.3$ & $(4-20)$ & 0.126 \\
\hline $\begin{array}{l}\text { Practice demand: preparedness } \\
\text { or scope of practice }\end{array}$ & $7.6 \pm 2.0$ & $(4-15)$ & $7.8 \pm 2.0$ & $(4-20)$ & 0.057 & $7.7 \pm 2.1$ & $(4-20)$ & $7.9 \pm 1.7$ & $(4-14)$ & 0.151 \\
\hline
\end{tabular}

Note: $p$ values calculated by $\chi^{2}$ test for nominal variables and Student's $t$-test for interval variables. $p$ values $<0.05$ are bolded. Samples vary due to missing cases: Online/electronic education frequent use $(n=249-270)$ and infrequent use $(n=753-841)$; Internet search engines frequent use $(n=660-709)$ and infrequent use $(n=340-401)$. 
support resources, and research databases. The use of print sources followed a similar pattern, with nurses reporting most frequent use of policies or protocols, followed by clinical practice guidelines, textbooks, and journals.

A report from the larger RRNII study indicates that following protocols or decision support tools to arrive at a plan of care is among the top four practice responsibilities that rural and remote RNs and LPNs reported (ranked by frequency) [17]. This suggests that the use of policies or protocols and clinical practice guidelines is an integral part of practice for both nurse groups. The only information source used more often than online policies or protocols by both nurse groups, according to the present study, was Internet searching.

The top ranking of Internet search engines was similarly found in a study of newly graduated LPNs in one Canadian province [54]. Where rural nurses work alone or with few colleagues [55, 56], an Internet search may be an accessible alternative to consulting a colleague. An Internet search can offer information that is relevant to rural practice in a convenient and more timely manner than a search of online/electronic textbooks, journals, and databases. Furthermore, research databases and academic journals that require subscriptions might not be widely available to many rural nurses. Nevertheless, the low use of online/electronic practice support resources and research databases in our results, relative to higher use of Internet search engines such as Google, suggests that rural and remote nurses are accessing non-peer-reviewed information of questionable quality to a greater extent than research-based information. Evidence suggests that the main barriers to using research literature (e.g., electronic database subscriptions) among nurses include time, skill, and access [57].

While RN and LPN similarities were apparent in our research, distinct differences also emerged. First, RNs were more likely than LPNs to report direct workplace access to all communication tools, except for high-speed Internet, which was equally accessible to both groups. Second, RNs more often than LPNs drew on online education to update their nursing knowledge, as well as online and print information to make decisions in nursing practice (except for textbooks). Among LPNs who were infrequent users compared to frequent users of online/electronic education to update their nursing knowledge, we found perceptions of lower use of resources related to training/professional development and technology (e.g., access to electronic resources).

These findings correspond to evidence suggesting that some new LPN graduates, the majority of whom hold two-year diplomas, have not been well prepared to identify, access, and evaluate the most appropriate research for practice [54]. The expectation that LPNs will use research evidence to the same extent as RNs in nursing practice varies across Canadian LPN regulatory groups [10]. Our findings point to a need for future research on the continuing education and information needs of LPNs and RNs relative to their positions and workplaces, particularly in rural and remote communities where nurses may work alone or with few colleagues to consult. Given their different educational requirements and practice responsibilities, further examination of LPN and RN preparation and capacity to apply new knowledge in practice is also warranted.

For nurses in this study, direct access to electronic communication among providers and teleconference and videoconference tools were lowest in long-term care practice settings. Long-term care practice settings also demonstrated the lowest use of online/electronic and in-person education to update nursing knowledge and the lowest use of online/electronic information to make specific decisions in nursing practice. A study of rural and urban long-term care facilities in a Canadian province found that access and incentives to take part in continuing education were important enablers, but the success of continuing education programs was largely determined by organizational support such as adequate resources and opportunities to subsequently implement new care initiatives [56]. Stolee et al. emphasize that although training is necessary, it is not sufficient to improve patient care [58]. Staff must feel empowered to change practice, and the workplace must share a common goal of quality improvement. Further research to investigate variations in the use and uptake of education and information across rural health care practice settings is merited.

The results of our research suggest that work engagement and technology availability in the workplace are important correlates of education and information use among rural and remote nurses. It is possible that information technology availability 
and workplace engagement are associated; however, we did not examine this relationship in the present analysis. Others have noted the important role of rural workplaces in facilitating research use by providing technology, including computers and Internet access, as well as time to search for information and take part in continuing education programs $[24,25,34]$. Williams further suggests that information technology may reduce professional isolation and, in turn, improve retention of rural nurses [31].

\section{Limitations}

This analysis has some limitations that should be noted. Our sample was representative of rural Canadian RNs and LPNs [17, 59]; however, our findings might not be generalizable to rural nurses outside of Canada. The survey was administered in 2014-2015, and changes in technology since that time should be taken into account. Survey respondents estimated the frequency of their education and information use; however, selfreported data are vulnerable to recall bias and social desirability bias [60]. Individuals who complete surveys may have a more positive attitude toward research; therefore, our findings may overrepresent rural and remote nurses who use education and information, and overestimate the use of such sources. Also, while this analysis assessed the frequency with which certain sources of information were used, the nature and quality of the information applied in practice was not evaluated.

\section{CONCLUSIONS}

Opportunities for rural and remote nurses to participate in and apply knowledge from continuing education and ongoing information use are important for safe and effective patient care. However, it is equally important that nurses critically reflect on new knowledge and, as much as possible, apply knowledge that is research-based. Future investigations could yield further insights into the specific forms of online/electronic information and education topics that are available to rural and remote nurses, and should explore how nurses in these communities evaluate and select the information that they ultimately use in practice. Moreover, further research about the factors associated with variations in education and information use according to registration status and practice setting would be valuable.

\section{ACKNOWLEDGMENTS}

The article stems from the study, "Nursing Practice in Rural and Remote Canada II," led by Martha MacLeod, Norma Stewart, and Judith Kulig. We are grateful to the nurses who responded to this survey and the advisory team led by Penny Anguish. We also thank Leana Garraway, Nadine Mix, and Janna Olynick for their contributions.

\section{FINANCIAL SUPPORT}

The Nursing Practice in Rural and Remote Canada II study was funded by the Canadian Institutes of Health Research (MOP 130260).

\section{REFERENCES}

1. Canadian Institute for Health Information. Regulated nurses, 2017: Canada and jurisdictional highlights [Internet]. The Institute; 2017 [cited 6 Nov 2018]. <https://www.cihi.ca/sites/default/files/document/regu lated-nurses-2017-pt-highlights-en-web.pdf>.

2. US Department of Health and Human Services, Health Resources and Services Administration, Bureau of Health Workforce, National Center for Health Workforce Analysis. Supply and demand projections of the nursing workforce: 2014-2030 [Internet]. The Department; 2017 [cited 9 May 2019]. <https://bhw.hrsa.gov/sites/default/files/bhw/nchwa /projections/NCHWA_HRSA_Nursing_Report.pdf $>$.

3. National Council of State Boards of Nursing. About US boards of nursing [Internet]. The Boards; 2019 [cited 9 May 2019]. <https://www.ncsbn.org/about-boards-ofnursing.htm $>$.

4. Canadian Nurses Association. The licensing exam [Internet]. The Association; 2019 [cited 9 May 2019]. $<$ https:// www.cna-aiic.ca/en/nursing-practice/nursingas-a-career/the-licensing-exam>.

5. Canadian Nurses Association. Regulation of RNs [Internet]. The Association; 2019 [cited 9 May 2019]. <https://cnaaiic.ca/en/nursing-practice/the-practice-ofnursing/regulation-of-rns $>$.

6. Canadian Council for Practical Nurses Regulators. Registering to be a LPN in Canada [Internet]. The Council; 2019 [cited 9 May 2019]. <http:/ / www.ccpnr.ca/ becomean-lpnrpn/>.

7. Canadian Practical Nurses Association. History of Canadian Practical Nurses Association [Internet]. The Association; 2003 [cited 9 May 2019].

$<$ http://www.lpnabc.ca/wp-content/uploads/cpnahistory.pdf $>$.

8. Canadian Nurses Association. Becoming an RN [Internet]. The Association; 2018 [cited 6 Nov 2018]. <https:// cnaaiic.ca/en/nursing-practice/the-practice-ofnursing/becoming-an-rn>. 
9. Canadian Nurses Association. $\mathrm{RN}$ and baccalaureate education [Internet]. The Association; 2018 [cited 6 Nov 2018]. < https:// www.cna-aiic.ca/en/nursing-practice/thepractice-of-nursing/education/rn-baccalaureateeducation>.

10. Butcher DL, MacKinnon KA. Educational silos in nursing education: a critical review of practical nurse education in Canada. Nurs Inq. 2015 Sep;22(3): 231-9.

11. Squires J, Baumbusch J, Demery Varin M, MacDonald I, Chamberlain S, Bostrom A, Thompson G, Cummings G, Estabrooks C. A profile of regulated nurses employed in Canadian long-term care facilities. Can J Aging. 2019;38(2):130-42.

12. Limoges J, Jagos K. The influences of nursing education on the socialization and professional working relationships of Canadian practical and degree nursing students: a critical analysis. Nurse Educ Today. 2015 Oct;35(10):1023-7.

13. Nurses Association of New Brunswick. Guidelines for intraprofessional collaboration: registered nurses and licensed practical nurses working together [Internet]. The Association; 2015 [cited 9 May 2019].

$<$ http://www.nanb.nb.ca/media/resource/NANBGuidelinesIntraprofessionalCollaborationRNsandLPNsWor kingTogether-E-2015-10.pdf $>$.

14. Canadian Institute for Health Information. Regulated nurses, 2016: registered nurse/nurse practitioner data tables [Internet]. The Institute; 2017 [cited 6 Nov 2018]. <https:// www.cihi.ca/en/regulated-nurses-2016registered-nursenurse-practitioner-data-tables $>$.

15. Canadian Institute for Health Information. Regulated nurses, 2016: licensed practical nurse data tables [Internet]. The Institute; 2017 [cited 6 Nov 2018]. <https:/ / www.cihi.ca/en/regulated-nurses-2016-licensedpractical-nurse-data-tables $>$.

16. Statistics Canada. Population by broad age groups and sex, for both sexes, Canada, by statistical area classification, 2016 census [Internet]. Statistics Canada; 2017 [cited 18 Jul 2019]. <https:/ / www12.statcan.gc.ca/censusrecensement/2016/dp-pd/hlt-fst/as/index-eng.cfm>

17. MacLeod M, Stewart N, Kulig J, Olynick J, Jónatansdóttir S, Kosteniuk J. Registered nurse survey final report: nursing practice in rural and remote Canada. In: Nursing practice in rural and remote Canada II. RRN2-04-01.1 [Internet]. Prince George, BC: University of Northern British Columbia; 2017 [cited 18 Jul 2019]. $<$ https://www.unbc.ca/sites/default/files/sections/rur al-nursing/en/rrniirnfinalreportupdated20190312.pdf>.

18. Barrett A, Terry DR, Quynh L, Hoang H. Factors influencing community nursing roles and health service provision in rural areas: a review of literature. Contemp Nurse. 2016 Jun;52(1):119-35.

19. Macleod M, Martin Misener R, Banks K, Morton AM, Vogt C, Bentham D. "I'm a different kind of nurse": advice from nurses in rural and remote Canada. Nurs Leadersh. 2008 Sep;21(3):40-53.

20. Ford D. Four persistent rural healthcare challenges. Healthc Manage Forum. 2016 Nov;29(6):243-6.
21. Malatzky C, Bourke L. Re-producing rural health: challenging dominant discourses and the manifestation of power. J Rural Stud. 2016 Jun;45:157-64.

22. Mitton C, Dionne F, Masucci L, Wong S, Law S. Innovations in health service organization and delivery in northern rural and remote regions: a review of the literature. Int J Circumpolar Health. 2012 Mar;70(5):460-72.

23. Fahs $P$. Leading-following in the context of rural nursing. Nurs Sci Q. 2017 Apr;30(2):176-8.

24. Winters CA, Lee HJ, Besel J, Strand A, Echeverri R, Jorgensen KP, Dea JE. Access to and use of research by rural nurses. Rural Remote Health. 2007 Aug;7:758.

25. Hendrickx L, Winters C. Access to continuing education for critical care nurses in rural or remote settings. Crit Care Nurs. 2017 Apr;37(2):66-71.

26. MacLeod M, Place J. Rural-focused nursing education: a summative evaluation of RNs' experiences of the rural nursing certificate program. Qual Adv Nurs Educ. 2015 Jun;1(2):article 3.

27. Penz K, D'Arcy C, Stewart N, Kosteniuk J, Morgan D, Smith B. Barriers to participation in continuing education activities among rural and remote nurses. J Contin Educ Nurs. 2007 Mar/Apr;38(2):58-66.

28. Place J, MacLeod M, John N, Adamack M, Lindsey AE. "Finding my own time": examining the spatially produced experiences of rural RNs in the rural nursing certificate program. Nurs Educ Today. 2012 Jul;32(5):581-7.

29. Olade R. Evidence-based practice and research utilization activities among rural nurses. J Nurs Scholarsh. 2004 Sep;36(3):220-5.

30. Fairchild RM, Everly M, Walters L, Bauer R, Laws S, Anderson L. Rural nurses' continuing education needs: a U.S. multi-site survey reveals challenges and opportunities. J Nurse Educ Pract. 2013 May;3(5):45-55.

31. Williams MA. Rural professional isolation: an integrative review. J Rural Nurs Health Care. 2012 Apr;12(2):3-10.

32. Canadian Radio-Television and Telecommunications Commission. CRTC Submission to the Government of Canada's Innovation Agenda December 21, 2016 [Internet]. The Commission; 2016 [cited 6 Nov 2018]. <https://crtc.gc.ca/eng/publications/reports/rp161221/r p161221.htm>.

33. Federal Communications Commission. 2016 broadband progress report [Internet]. The Commission; 2016 [cited 9 May 2019]. <https://www.fcc.gov/reportsresearch/reports/broadband-progress-reports/2016broadband-progress-report>.

34. O'Lynn C, Luparell S, Winters CA, Shreffler-Grant J, Lee HJ, Hendrickx L. Rural nurses' research use. Online J Rural Nurs Health Care. 2009 Jun;9(1):34-45.

35. Jukkala AM, Henly SJ, Lindeke LL. Rural perceptions of continuing professional education. J Contin Educ Nurs. 2008 Dec;39(12):555-63. 
36. Koessel BD, Winters CA, Lee HJ, Hendrickx L. Rural nurses' attitudes and beliefs toward evidence-based practice. In: Winters C, ed. Rural nursing: concepts, theory, and practice. 4th ed. New York, NY: Springer Publishing; 2013. p. 275-91.

37. Carter-Templeton $\mathrm{HD}, \mathrm{Wu} \mathrm{L}$. Using mobile technologies to access evidence-based resources: a rural health clinic experience. Nurs Clin N Am. 2015 Sep;50(3):596-603.

38. Hodge AJ, Miller EL, Dilts Skaggs MK. Nursing selfperceptions of emergency preparedness at a rural hospital. J Emerg Nurs. 2017 Jan;43(1):10-4.

39. Mills J, Field J, Cant R. Rural and remote Australian general practice nurses' sources of evidence for knowledge translation: a cross-sectional survey. Int J Evid Based Healthc. 2011 Sep;9:246-51.

40. Ortiz J, Bushy A. A focus group study of rural health clinic performance. Fam Community Health. 2011 Apr/Jun;34(2):111-8.

41. Koschel A, Cross M, Haines H, Ervin K, Skinner-Louis D, Carbone D. Research and evidence based practice in a rural Victorian cohort. Aust J Adv Nurs. 2013 Dec;30(2):13-9.

42. Stewart NJ, D'Arcy C, Pitblado JR, Morgan DG, Forbes D, Remus G, Smith B, Andrews ME, Kosteniuk J, Kulig JC, MacLeod ML. A profile of registered nurses in rural and remote Canada. Can J Nurs Res. 2005 Mar;37(1):122-45.

43. MacLeod MLP, Stewart NJ, Kulig JC, Anguish P, Andrews ME, Banner D , Garraway L, Hanlon N, Karunanayake C, Kilpatrick K, Koren I, Kosteniuk J, Martin-Misener R, Mix N, Moffitt P, Olynick J, Penz K, Sluggett L, Van Pelt L, Wilson E, Zimmer L. Nurses who work in rural and remote communities in Canada: a national survey. Hum Resour Health. 2017 May;15(1):34.

44. du Plessis V, Beshiri R, Bollman RD, Clemenson H. Definitions of rural. Rural Small Town Can Anal Bull. 2001 Nov;3(3):1-17.

45. Dillman DA, Smyth JD, Christian LM. Internet, phone, mail and mixed-mode surveys: the tailored design method. 4th ed. Hoboken, NJ: Wiley \& Sons; 2014.

46. Marshall AP, West SH, Aitken LM. Preferred information sources for clinical decision making: critical care nurses' perceptions of information accessibility and usefulness. Worldviews Evid Based Nurs. 2011 Dec;8(4):224-5.

47. Gilmour JA, Huntington A, Broadbent R, Strong A, Hawkins M. Nurses' use of online health information in medical words. J Adv Nurs. 2012 Jun;68(6):1349-58.

48. O'Leary DF, Mhaolrúnaigh SN. Information-seeking behaviour of nurses: where is information sought and what processes are followed? J Adv Nurs. 2012 Feb;58(2):379-90.

49. Schaufeli WB, Bakker AB, Salanova M. The measurement of work engagement with a short questionnaire: a crossnational study. Educ Psychol Meas. 2006 Aug;66(4):701-16.

50. Meyer JP, Allen NJ, Smith CA. Commitment to organizations and occupations: extension and test of a three-component conceptualization. J Appl Psychol. 1993 Aug;78(4):538-51.
51. Penz K, Kosteniuk J, Stewart N, MacLeod M, Kulig J, Karunanayake C, Kilpatrick K. Development and psychometric evaluation of the Job Demands in Nursing (JDIN) scale and Job Resources in Nursing (JRIN) scale: results from a national study. Nurs Open. 2018 Nov;6(2):348-366.

52. Carter L, Hanna M, Warry W. Perceptions of the impact of online learning as a distance-based learning model on the professional practices of working nurses in northern Ontario. Can J Learn Technol. 2016 Summer;42(3):1-15.

53. Rohatinsky NK, Jahner S. Supporting nurses' transition to rural healthcare environments through mentorship. Rural Remote Health. 2016 Jan-Mar;16(1):3637.

54. Wadson K, Phillips LA. Information literacy skills and training of licensed practical nurses in Alberta, Canada: results of a survey. Health Inf Libr J. 2018 Jun;35(2):141-59.

55. Andrews ME, Stewart NJ, Pitblado JR, Morgan DG, Forbes $\mathrm{D}, \mathrm{D}^{\prime}$ Arcy C. Registered nurses working alone in rural and remote Canada. Can J Nurs Res. 2005 Mar;37(1):14-33.

56. Hunsberger M, Baumann A, Blythe J, Crea M. Sustaining the rural workforce: nursing perspectives on worklife challenges. J Rural Health. 2009 Dec;25(1):17-25.

57. Sadoughi F, Azadi T, Azadi T. Barriers to using electronic evidence based literature in nursing practice: a systematised review. Health Inf Libr J. 2017 Sep;34(3):18799.

58. Stolee P, Esbaugh J, Aylward S, Cathers T, Harvey D, Hillier LM, Keat N, Feightner JW. Factors associated with the effectiveness of continuing education in long-term care. Gerontologist. 2005 Jun;45(3):399-405.

59. MacLeod M, Stewart N, Kulig J, Olynick J, Kosteniuk J, Jonatansdottir S. Licensed/registered practical nurse national survey report: nursing practice in rural and remote Canada. In: Nursing practice in rural and remote Canada II. RRN2-04-02 [Internet]. Prince George, BC: University of Northern British Columbia; 2017 [cited 17 Jul 2019]. <https://www.unbc.ca/sites/default/files/sections/ruralnursing/en/rrniilpnfinalreportupdated20190131.pdf>.

60. Althubaiti A. Information bias in health research: definition, pitfalls, and adjustment methods. J Multidiscip Healthc. 2016 May 4;9:211-7.

\section{SUPPLEMENTAL FILE}

- Appendix: Survey information and education sources questions 


\section{AUTHORS' AFFILIATIONS}

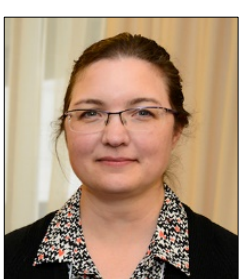

\section{Julie Kosteniuk, PhD,}

julie.kosteniuk@usask.ca, Professional Research Associate, Canadian Centre for Health and Safety in Agriculture, College of Medicine, University of Saskatchewan, Saskatoon, SK, Canada

Norma J. Stewart, PhD, RN,

norma.stewart@usask.ca, Professor Emerita, College of Nursing, University of

Saskatchewan, Saskatoon, SK, Canada

Erin C. Wilson, PhD, NP(F), erin.wilson@unbc.ca, Assistant Professor, School of Nursing, University of Northern British Columbia, Prince George, BC, Canada

Kelly L. Penz, PhD, RN, kelly.penz@usask.ca, Assistant Professor, College of Nursing, University of Saskatchewan, Regina Campus, Regina, SK, Canada

Ruth Martin-Misener, PhD, NP, ruth.martin-misener@dal.ca, Professor, School of Nursing, Dalhousie University, Halifax, NS, Canada
Debra G. Morgan, PhD, RN, debra.morgan@usask.ca, Professor, Canadian Centre for Health and Safety in Agriculture, College of Medicine, University of Saskatchewan, Saskatoon, SK, Canada

Chandima Karunanayake, PhD, chandima.karunanayake@usask.ca, Professional Research Associate, Canadian Centre for Health and Safety in Agriculture, College of Medicine, University of Saskatchewan, Saskatoon, SK, Canada

Martha L. P. MacLeod, PhD, RN, macleod@unbc.ca, Professor and Northern Health - UNBC Knowledge Mobilization Research Chair, School of Nursing, University of Northern British Columbia, Prince George, BC, Canada

Received November 2018; accepted June 2019 (c) (i) Articles in this journal are licensed under a $\underline{\text { Creative }}$ Commons Attribution 4.0 International License.

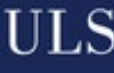

This journal is published by the University Library System of the University of Pittsburgh as part of its D-Scribe Digital Publishing Program and is cosponsored by the University of Pittsburgh Press. 